\title{
Adaptación, validación y puntos de corte del Cuestionario de edades y etapas-3ra edición (ASQ-3) en español, en una zona urbana de Lima-Perú.
}

Adaptation, validation and cut-off values of the Ages \& Stages questionnaire 3th edition (ASQ-3) Spanish version in a Lima, Perú urban setting.

Adriel Gudiel-Hermoza ${ }^{1, a}$, Jorge Gudiel-Hermoza ${ }^{1, a}$, Daniel Guillén-Pinto 2,b

\section{RESUMEN}

Objetivo: Adaptar y determinar la confiabilidad, validez y puntos de corte de la versión en español del Cuestionario de edades y etapas tercera edición (ASQ-3), un instrumento de vigilancia y tamizaje del desarrollo psicomotor, en una población urbana de la zona norte de Lima, Perú. Material y Métodos: Mediante muestreo por conveniencia, se aplicaron formas del cuestionario ASQ-3 a padres de niños entre 1 a 66 meses de edad, en un entorno de consulta ambulatoria. Resultados: Se aplicó un total de 5,830 cuestionarios correspondientes a 3,570 niños, 2,667 (45,7 \%) de los cuales contaban entre 14 y 36 meses de edad. La consistencia interna general y por cada área del desarrollo, evaluadas mediante el Índice alfa ordinal, oscilaron entre 0,70 - 0,94. El Índice de validez de contenido por el criterio de jueces, medido con el método Lawshe, la $\mathrm{V}$ de Aiken o ambos, fue válido para la mayoría de los cuestionarios. La correlación biserial ítem-test fue $>0,30$ en la mayoría de los ítems $(p<0,010)$. El Índice error medio cuadrático de aproximación (RMSEA) del análisis factorial fue $<0.10$, puntualizando un nivel adecuado para las cinco áreas de evaluación del ASQ-3. Los puntos de corte fueron determinados por intervalos de edad y área. Conclusiones: La versión en español del ASQ-3 adaptada a la población del estudio demostró ser un instrumento confiable y válido para la vigilancia y tamizaje del desarrollo psicomotor de niños menores de 5 años de edad en un entorno urbano de consulta ambulatoria en Lima, Perú.

PALABRAS CLAVE: ASQ-3, niños, desarrollo psicomotor, validez, confiabilidad.

\section{SUMMARY}

Objective: A cultural-linguistic adaptation of the Age and Stages Questionnaire third edition (ASQ-3)-Spanish version was performed in order to determine its reliability, validity and cut-off points in an urban population and pediatric outpatient setting of Lima, Perú. Material and Methods: Questionnaire forms were applied to parents of children between 1 to 66 months old of age, chosen through a convenience sampling method. Results: A total of 5,830 questionnaires were applied to parents of 3,507 children, 2667 (45.7\%) of whom had between 14 and 36 months of age. The general internal consistency and that of five specific areas, measured by means of the Ordinary alpha index oscillated between 0.70-0.94. Content validity by judges' criteria, using the index by Lawshe method, the Aiken V coefficient, or both, was valid for most of the questionnaires. The biserial item-test correlations were

Clínica Mundo Salud. Lima, Perú.

Universidad Peruana Cayetano Heredia. Lima, Perú.

a Pediatra; ${ }^{\text {NNeuropediatra }}$ 
$>0.30$ for most items $(\mathrm{p}<0.010)$. The construct validity according to the factorial analysis, determined that the five factors or areas of the ASQ-3 were adequate. Cut-off points were determined by age and area intervals of the ASQ3. Conclusions: The Spanish version of the ASQ-3 proved to be a reliable and valid tool to survey and screen the psychomotor development of children under 5 years of age in an urban outpatient area setting of Lima, Perú.

KEY WORDS: ASQ-3, children, psychomotor development, validity, reliability.

\section{INTRODUCCIÓN}

El desarrollo psicomotor del niño suele transcurrir de manera predecible o típica; sin embargo, $\sim 15 \%$ de los niños menores de 5 años podrían presentar alteraciones en el neurodesarrollo (1-3). La sensibilidad del clínico para identificar estas alteraciones suele ser $\sim 30 \%$, por lo que se recomienda el uso de instrumentos estandarizados y validados que aumentaría en 2 a 6 veces su capacidad de detección $(4,5)$.

El Cuestionario de edades y etapas- tercera edición (ASQ-3) versión en español (6), es un cuestionario de vigilancia del desarrollo psicomotor de banda ancha completado por los padres. Evalúa 5 áreas del neurodesarrollo: Comunicación, motora gruesa, motora fina, resolución de problemas y Socialindividual. Siendo 21 cuestionarios por edades y etapas con 6 ítems por área y 3 posibles respuestas tipo Likert cada uno. El puntaje alcanzado determinará la zona de calificación: "Desarrollo típico", "Necesidad de monitoreo" o "Necesidad de una evaluación adicional".

Si bien la falta de tiempo, reembolsos, familiaridad con los instrumentos o disponibilidad de los servicios podrían ser barreras en la implementación y aplicación de las pruebas de detección, su uso cotidiano por ejemplo entre los pediatras de Norteamérica, se triplicó de 23\% el 2002 a 63\% el 2016 (7). El Comité de la Infancia de la Academia americana de pediatría (AAP) y "Bright futures" recomiendan realizar vigilancia y tamizaje del desarrollo de manera estructurada y sistematizada con el uso de herramientas estandarizadas y validadas a los 9,18, y 24 o 30 meses de edad $(8,9)$.

El "Seminario sobre medición del desarrollo infantil", organizado por Diálogo Interamericano, el Grupo de Análisis para el Desarrollo y el Banco Mundial, llevado a cabo en Lima, Perú-2016; invocaron la necesidad de "impulsar o fortalecer la medición sistemática con representatividad poblacional del desarrollo infantil, así como de cuáles instrumentos utilizar y su implementación a escala en los países de la región" (10).

En la práctica de consulta ambulatoria de pediatría en zona urbana de la ciudad de Lima no se cuenta con instrumentos de vigilancia del desarrollo psicomotor de banda ancha. Se requiere del uso de Cuestionarios completados por los padres que faciliten la viabilidad en su aplicación y estén adaptados culturallingüísticamente, validados y con puntos de corte propios.

Actualmente el ASQ-3 es el instrumento de vigilancia del desarrollo psicomotor más empleado en los EE.UU. (7-9), lugar donde se validó originalmente $(11,12)$.

El ASQ-3 está conformado por 21 cuestionarios con intervalos de edad de 2 a 60 meses, en su segunda y tercera edición se encuentra traducido al español en su lugar de origen (EE.UU.), posee una buena confiabilidad mediante el análisis de consistencia interna, la validez de contenido y constructo son buenas, sensibilidad: 0,8 , especificidad: 0,85 y los puntos de corte establecidos para los 21 cuestionarios $(6,11,12)$. En Latinoamérica, la versión en español se encuentra validada en México (13), Brasil (14), Colombia (15), Chile (16) y Argentina (17).

En el Perú hay un estudio de validación del ASQ3 para algunas zonas rurales (18) y otro aplicado a la zona rural y urbana de la ciudad de Ayacucho y Lima (19), este último realizado con insuficiente tamaño muestral. En ambos casos no son aplicables para la estructura urbana y de consulta ambulatoria pediátrica local.

El objetivo del presente trabajo fue adaptar y determinar la confiabilidad, validez y puntos de corte de la versión en español del Cuestionario de edades $y$ etapas tercera edición (ASQ-3), un instrumento de vigilancia y tamizaje del desarrollo psicomotor, en una población urbana de la zona norte de Lima, Perú. 
Adaptación, validación y puntos de corte del Cuestionario de edades y etapas-3ra edición (ASQ-3) en español Lima-Perú.

\section{MATERIAL Y MÉTODOS}

Con la finalidad de validar y establecer puntos de corte de la versión en español del Cuestionario de Edades y etapas 3ra edición (ASQ-3) versión en español (6), se llevó a cabo el presente estudio en una Clínica particular de la zona urbana de la ciudad de Lima, población socioeconómica media.

Población objetivo: Padres de niños menores de 5 años de edad que acudieron a consulta externa por controles rutinarios o de niño sano, siendo el muestreo por conveniencia en el periodo de setiembre 2016 a febrero 2019. En los primeros 2 años del estudio se estuvo aplicando los cuestionarios de 2 a 36 meses, incluyéndose luego hasta los 60 meses.

Criterios de inclusión: Ser padres de familia con nivel de educación mínimo de 5to de primaria, cuyos niños estuvieron entre las edades de 1 mes 0 días a 66 meses 0 días. Acudir con sus niños por consulta ambulatoria del servicio de pediatría sea por controles rutinarios, aplicación de vacunas o control de niño sano. Algunos padres pudieron haber completado cuestionarios en diferentes edades de sus niños.

Criterios de exclusión: Cuestionarios con información incompleta. Niños con trastornos severos del desarrollo, anomalías genéticas o malformaciones congénitas múltiples. Presentar afecciones médicas de tipo agudo o crónico de grado moderado a severo y/o requieran hospitalización.

Instrumento: Se empleó la versión en español del Cuestionario de edades y etapas 3ra edición-2009 $(6,11)$, un instrumento estructurado cuyo constructo fue desarrollado para el proceso de vigilancia y tamizaje del desarrollo psicomotor de niños entre 1 a 66 meses de edad y establecer o no riesgo para ser referidos a mayor evaluación. También útil en poblaciones de alto riesgo biológico como la prematuridad. Es completado por padres o cuidadores con nivel educativo mínimo 5to de primaria. Requiere un tiempo de llenado entre 15 a 20 minutos y puntuación de 2 a 3 minutos. Consta de 21 cuestionarios, 12 en intervalos de 2 meses (2-24 meses), 4 en intervalos de 3 meses (24 - 36 meses) y 4 en intervalos de 6 meses (42- 60 meses). Desarrollado en inglés y traducido al español y francés, actualmente estandarizado, validado y adaptado cultural y lingüísticamente a muchos países del mundo. Evalúa 5 áreas del desarrollo del niño: Comunicación, motora gruesa, motora fina, resolución de problemas y socio-individual. Cada área consta de 6 ítems, 3 respuestas para cada uno: "Sí", "A veces" y "Aún no", con puntajes de 10, 5, 0, respectivamente. Se consigue un puntaje máximo de 60 por área. Los niveles de riesgo se establecen como: "Desarrollo típico", "Necesidad de monitoreo" o "Necesidad de una evaluación adicional". De sus propiedades psicométricas, su confiabilidad de test-retest 0,92, confiabilidad inter-operador 0,93 , consistencia interna con alfa de 0,51 a 0,87 ; aceptable a buena validez de contenido, sensibilidad 0,86, especificidad 0,85, concordancia con otros instrumentos estandarizados $0,86(11,12)$ y aceptable a buena validez concurrente con pruebas como el Inventario de desarrollo Battelle y la Escala Bayley de desarrollo infantil; útil además para el seguimiento de prematuros (20-24). También se ha descrito que podría tener alguna correlación con pruebas desarrolladas para la detección del autismo (25).

Adaptación lingüistica y cultural: Para su segunda edición el ASQ fue traducido al español y revisado por expertos en pediatría y pediatría del desarrollo y profesionales que trabajaban con niños y familias de habla hispana en diferentes dialectos (nicaragüense, mexicano, argentino); si bien el análisis del ASQ3 indica similares puntos de corte de riesgo entre la versión en inglés y español, se sugiere realizar una adaptación cultural y lingüística propias en cada grupo de población de habla hispana con la respectiva determinación de los puntos de corte de riesgo. Sin embargo, se recomienda realizar una adaptación lingüística y cultural para la población a aplicar la prueba. Para ello se siguió las pautas de adaptación cultural y lingüística dadas por la autora y Editorial del manual del ASQ-3 (26). Inicialmente se convocó a 3 psicólogos clínicos infantiles y un pediatra con dominio y experiencia en el uso del ASQ-3 versión en español, quienes realizaron las adaptaciones lingüísticas y culturales para cada uno de los 21 cuestionarios. Durante un periodo de 3 meses se hizo las modificaciones sugeridas con retroalimentación de su uso en la práctica hasta quedar una versión final. Por ejemplo, para el cuestionario de 6 meses, área de comunicación, ítem 5 según el cuestionario original indicaba: "¿Hace su bebé sonidos como "da", "ga”, "ka” y "ba”?”. Se modificó por: “¿Su bebé emite sonidos como "ma", "pa", "da", "ga", "ka" y "ba", u otra silaba?", debido a que en nuestro medio las primeras sílabas mencionadas por el bebé para esa edad son "ma", "pa", al referirse a mamá o papá, en lugar de "da" en referencia a Dad (papá en inglés). 
Procedimiento: Los cuestionarios fueron aplicados a los padres en la sala de espera del servicio de pediatría del establecimiento previa a la consulta con el pediatra. Si sus niños tuvieron el antecedente de prematuridad se hizo corrección de la edad gestacional, hasta los 24 meses de edad cronológica. Se dieron instrucciones sobre el correcto llenado del cuestionario proporcionándose un lápiz, tablero y se asignó un espacio en la sala de espera para su llenado. El tiempo asignado para ello fue de 15 a 20 minutos. Luego de verificar el correcto llenado se procedió a asignar los puntajes respectivos a cargo del personal de enfermería. Luego, el cuestionario se adjuntó para la revisión del pediatra en la consulta. Una vez allí, según los puntajes y puntos de corte hallados se estableció si el niño se encuentra o no en riesgo de someter a evaluación, esta vez a cargo del equipo del establecimiento conformado por psicología infantil, terapia del lenguaje, audiología y pediatra capacitado para llegar a un diagnóstico si correspondiera.

Análisis Estadístico: Teniendo el ASQ-3 original tres posibles respuestas tipo likert por ítem: "Sí", "A veces" y "Aún no", para efectos del análisis estadístico en el presente estudio se dicotomizó los 30 ítems de cada cuestionario en 2 respuestas de tipo ordinal: "Sí" y "Aún no"; debido a la poca frecuencia de respuestas tipo "a veces" que no permitió un análisis adecuado.

Confiabilidad: Se determinó la Consistencia interna mediante el Coeficiente alfa ordinal ( $\alpha$ ) (27), basado en una matriz de correlación tetracóricas para variables de respuesta binaria en lugar del Alfa de Cronbach. Se consideró adecuada si el $\alpha \geq 0,70$.

Validez de contenido. Criterio de jueces: Intervinieron 5 jueces. Se calificó 3 atributos: ("Suficiencia", "Pertinencia "y "Claridad") por ítem y cuestionario, calificando del 1 al 4 . Por ejemplo, en "suficiencia": 1= Insuficiente, 2= Poco suficiente, 3= Medianamente suficiente, $4=$ Suficiente, y de la misma manera para los atributos pertinencia y claridad (ver Anexo 2). Para su análisis se aplicó dos técnicas: a) La Razón de validez de contenido (RVC) propuesta por Lawshe C.H. (28) y mejorada por Tristán-López A. (29), para el caso en que el número de jueces fuera menor a 8. Un ítem fue válido si su RVC era $>0,5823$. b) El Coeficiente de validez V de Aiken (30), evaluó los 3 atributos en sus 4 categorías por ítem de los 21 cuestionarios. Se consideró que cualquier valor $\mathrm{V}$ de Aiken $<0,87$ no tendría significancia $(\mathrm{p}>0,05)$.

Validez de ítems: Debido a la conversión dicotómica de las respuestas: "Si" y "Aún no", se aplicó Correlaciones biseriales ( $r b$ ) de Spearman por el método Ítem-test entre la respuesta de cada ítem y el puntaje total por cuestionario. Un ítem fue considerado válido si $\mathrm{rb}>0,30$.

Validez de constructo: Para verificar si la estructura del ASQ-3 estuviera adecuadamente compuesta por 5 áreas o factores, se realizó Análisis factorial exploratorio (AFE) por el Método iterativo de factores principales (IPF) y Rotación oblimin. Previamente se verificó que cumplieran los requisitos para llevar a cabo un AFE. Después, se realizó el Análisis factorial confirmatorio $(A F C)$ mediante la Bondad de ajuste del modelo factorial para 5 dimensiones. Se consideró como adecuado el análisis factorial si el Índice error medio cuadrático de aproximación (RMSEA) fuese $<0,10$.

Puntos de corte y zonas de riesgo: Previos a la determinación de puntos de corte, definimos como "casos" , según los puntos de corte originales del ASQ-3, a los niños cuyos cuestionarios indicaron puntajes en la zona de "monitoreo" o "riesgo" de retraso psicomotor en una o varias áreas (>1 ó 2 D.E., respectivamente) y además por criterio clínico tener un diagnóstico específico (retraso de tipo motor grueso, motor fino o de la coordinación motora, retraso del lenguaje, retraso del desarrollo cognitivo, trastorno del espectro autista). Los "controles" se definieron como niños cuyos cuestionarios según el puntaje alcanzado se encontraban en el rango promedio o $<1$ D.E. y clínicamente sanos. En el presente estudio no se reportó los diagnósticos de los casos. Así, se tuvo 5728 cuestionarios asignados como "controles" y 102 como "casos". Para la determinación de los nuevos puntos de corte que establecieron las zonas de riesgo respectivas se empleó los estadísticos de media y desviación estándar (D.E.), según la metodología descrita en el manual del ASQ-3 (11,12). Los dos puntos de corte determinaron 3 zonas para establecer riesgo: Una zona "sin riesgo", con puntajes por encima de la media menos 1 D.E., una segunda zona de "monitoreo", con puntajes entre la media menos 1 D.E. y la media menos 2 D.E. y una tercera zona de "riesgo alto", con puntajes por debajo de 2 D.E. El análisis estadístico en general se realizó con el programa STATA.14.

Previo a su ejecución, el estudio fue revisado y aprobado por el Comité de Ética del establecimiento donde se llevó a cabo el estudio y se obtuvo el consentimiento de los padres para participar en el estudio de manera verbal. 
Adaptación, validación y puntos de corte del Cuestionario de edades y etapas-3ra edición (ASQ-3) en español Lima-Perú.

\section{RESULTADOS}

Se analizaron 5830 cuestionarios correspondientes a 3570 niños. De ellos, 3517 niños (5 728 cuestionarios) fueron considerados de niños sanos o "controles" y 53 niños (102 cuestionarios) como "casos". Según el género, $3132(53,7 \%)$ fueron del sexo masculino. En la tabla 1 se muestra la distribución porcentual de cuestionarios aplicados por intervalos de edad del cuestionario. El $94 \%$ de los cuestionarios aplicados correspondieron a $\leq 36$ meses. Cerca de la mitad de los cuestionarios 2667 (45,7\%), correspondieron a los grupos de edad comprendidos entre 14 y 36 meses. Análisis de confiabilidad: Mediante el análisis de consistencia interna, como se detalló en la tabla 2, para cada uno de los 21 cuestionarios, de manera general y para las cinco áreas, el Coeficiente alpha ordinal $(\alpha)$ fue de aceptable a muy buena $(0,70-0,94)$. En 11 de los 21 cuestionarios los valores fueron mayores a 0,90 . Del análisis por áreas para los cuestionarios entre 10 y 48 meses el $\alpha \geq 0,70$. La excepción fue para los 20 meses, área "resolución de problemas", el $\alpha=0,49$ y para 42 meses, área "motora gruesa", el $\alpha=0,20$.

Validez de contenido por Criterio de jueces: De la tabla 3 se observa que hubo validez de contenido para las 5 áreas en los 21 cuestionarios (IVC > 0,5823). Sin embargo, algunos ítems fueron menores de ese valor por haber desacuerdo entre los jueces. Los indicadores de validez de contenido de cada ítem en la

Tabla 1. Distribución porcentual de cuestionarios aplicados por intervalos de edad

\begin{tabular}{llllll}
\hline Edad (meses) & $\mathbf{2 - 1 2}$ & $\mathbf{1 4 - 2 4}$ & $\mathbf{2 7 - 3 6}$ & $\mathbf{4 2 - 4 8}$ & $\mathbf{5 4 - 6 0}$ \\
\hline Cuestionarios N=5 830 & 2817 & 1530 & 1137 & 217 & 129 \\
$(\%)$ & $(48,3)$ & $(26,2)$ & $(19,5)$ & $(3,7)$ & $(2,2)$ \\
\hline
\end{tabular}

Tabla 2. Consistencia Interna- Coeficiente Alfa ordinal del ASQ-3 por áreas e intervalos de edad

\begin{tabular}{lcccccccccccccccccccccccccccccc}
\hline Mes & $\mathbf{2}$ & $\mathbf{4}$ & $\mathbf{6}$ & $\mathbf{8}$ & $\mathbf{9}$ & $\mathbf{1 0}$ & $\mathbf{1 2}$ & $\mathbf{1 4}$ & $\mathbf{1 6}$ & $\mathbf{1 8}$ & $\mathbf{2 0}$ & $\mathbf{2 2}$ & $\mathbf{2 4}$ & $\mathbf{2 7}$ & $\mathbf{3 0}$ & $\mathbf{3 3}$ & $\mathbf{3 6}$ & $\mathbf{4 2}$ & $\mathbf{4 8}$ & $\mathbf{5 4}$ & $\mathbf{6 0}$ \\
\hline Comunicación & 0,814 & 0,676 & 0,591 & $-0,100$ & 0,793 & 0,778 & 0,709 & 0,756 & 0,839 & 0,878 & 0,868 & 0,865 & 0,920 & 0,885 & 0,903 & 0,895 & 0,800 & 0,669 & 0,847 & 0,925 & 0,337 & \\
Motor Gruesa & 0,730 & 0,722 & 0,750 & 0,846 & 0,892 & 0,932 & 0,916 & 0,942 & 0,797 & 0,745 & 0,754 & 0,694 & 0,829 & 0,780 & 0,739 & 0,889 & 0,788 & 0,208 & 0,739 & 0,700 & $-0,357$ \\
Motor Fina & 0,627 & 0,783 & 0,820 & 0,848 & 0,597 & 0,901 & 0,851 & 0,754 & 0,817 & 0,795 & 0,770 & 0,750 & 0,684 & 0,644 & 0,805 & 0,789 & 0,807 & 0,746 & 0,758 & 0,787 & 0,665 & \\
$\begin{array}{l}\text { Resolución de } \\
\text { Problemas }\end{array}$ & 0,864 & 0,823 & 0,800 & 0,266 & 0,783 & 0,784 & 0,801 & 0,819 & 0,828 & 0,817 & 0,495 & 0,770 & 0,697 & 0,703 & 0,831 & 0,844 & 0,786 & 0,756 & 0,769 & 0,221 & 0,314 \\
$\begin{array}{l}\text { Socio- } \\
\text { individual }\end{array}$ & 0,694 & 0,550 & 0,692 & 0,688 & 0,598 & 0,688 & 0,803 & 0,727 & 0,810 & 0,788 & 0,783 & 0,639 & 0,728 & 0,703 & 0,678 & 0,792 & 0,745 & 0,691 & 0,793 & 0,452 & 0,517 \\
General & 0,888 & 0,874 & 0,910 & 0,851 & 0,882 & 0,916 & 0,925 & 0,922 & 0,904 & 0,925 & 0,892 & 0,885 & 0,921 & 0,898 & 0,928 & 0,945 & 0,924 & 0,854 & 0,918 & 0,706 & 0,778
\end{tabular}

En la tabla se observa que el Índice alfa ordinal fue de aceptable a muy buena $(0,70-0,94)$, por grupo de edad y área del desarrollo.

Tabla 3. Índice de Validez de Contenido (IVC) del cuestionario ASQ3 por dimensiones

\begin{tabular}{|c|c|c|c|c|c|c|c|c|c|c|c|c|}
\hline Áreas & Ítems & $2 \mathrm{~m}$ & $4 \mathrm{~m}$ & $6 \mathrm{~m}$ & $8 \mathrm{~m}$ & $9 \mathrm{~m}$ & $10 \mathrm{~m}$ & $12 \mathrm{~m}$ & $14 \mathrm{~m}$ & $16 \mathrm{~m}$ & $18 \mathrm{~m}$ & $20 \mathrm{~m}$ \\
\hline Escala & & 0.907 & 0.860 & 0.893 & 0.913 & 0.913 & 0.940 & 0.927 & 0.947 & 0.933 & 0.933 & 0.940 \\
\hline Comunicación & 01 a 06 & 0.867 & 0.833 & 0.833 & 0.933 & 0.967 & 0.933 & 0.767 & 0.933 & 0.800 & 0.800 & 0.900 \\
\hline Motora gruesa & 07 a 12 & 0.800 & 0.767 & 0.933 & 0.967 & 0.933 & 0.967 & 1.000 & 0.967 & 0.967 & 1.000 & 1.000 \\
\hline Motora fina & 13 a 18 & 1.000 & 0.900 & 0.900 & 0.800 & 0.867 & 0.900 & 0.967 & 0.967 & 1.000 & 0.967 & 0.967 \\
\hline Resol. Problemas & 19 a 24 & 0.900 & 0.833 & 0.933 & 0.867 & 0.900 & 0.967 & 0.967 & 1.000 & 0.933 & 0.900 & 0.900 \\
\hline Socio-individual & 25 a 30 & 0.967 & 0.967 & 0.867 & 1.000 & 0.900 & 0.933 & 0.933 & 0.867 & 0.967 & 1.000 & 0.933 \\
\hline & Ítems & $22 \mathrm{~m}$ & $24 \mathrm{~m}$ & $27 \mathrm{~m}$ & $30 \mathrm{~m}$ & $33 \mathrm{~m}$ & $36 \mathrm{~m}$ & $42 \mathrm{~m}$ & $48 \mathrm{~m}$ & $54 \mathrm{~m}$ & $60 \mathrm{~m}$ & \\
\hline Escala & & 0.993 & 0.947 & 0.953 & 0.993 & 0.993 & 0.987 & 0.913 & 0.967 & 0.940 & 0.960 & \\
\hline Comunicación & 01 a 06 & 0.967 & 0.900 & 1.000 & 1.000 & 1.000 & 1.000 & 0.933 & 0.967 & 0.800 & 1.000 & \\
\hline Motora gruesa & 07 a 12 & 1.000 & 0.933 & 1.000 & 1.000 & 1.000 & 1.000 & 0.933 & 0.933 & 1.000 & 1.000 & \\
\hline Motora fina & 13 a 18 & 1.000 & 0.933 & 0.867 & 1.000 & 0.967 & 1.000 & 1.000 & 1.000 & 0.933 & 0.867 & \\
\hline Resol. problemas & 19 a 24 & 1.000 & 0.967 & 0.900 & 0.967 & 1.000 & 0.933 & 0.700 & 0.933 & 1.000 & 0.967 & \\
\hline Socio-individual & 25 a 30 & 1.000 & 1.000 & 1.000 & 1.000 & 1.000 & 1.000 & 1.000 & 1.000 & 0.967 & 0.967 & \\
\hline
\end{tabular}

M: Meses, Resol.: Resolución de problemas 
Gudiel-Hermoza A, et al.

Tabla 4. Estructura factorial del ASQ-3 y prueba de "Bondad de ajuste" según Índice RMSEA por intervalo de edad

\begin{tabular}{lcccccccccc}
\hline Mes & $\mathbf{2}$ & $\mathbf{4}$ & $\mathbf{6}$ & $\mathbf{8}$ & $\mathbf{9}$ & $\mathbf{1 0}$ & $\mathbf{1 2}$ & $\mathbf{1 4}$ & $\mathbf{1 6}$ & $\mathbf{1 8}$ \\
\hline RMSEA & 0,07 & 0,06 & 0,07 & 0,06 & 0,08 & 0,06 & 0,07 & 0,08 & 0,08 & 0,07 \\
Mes & 20 & 22 & 24 & 27 & 30 & 33 & 36 & $* 42$ & 48 & $* * 54$ \\
RMSEA & 0,07 & 0,06 & 0,07 & 0,07 & 0,08 & 0,09 & 0,06 & 0,10 & 0,10 & 0,10
\end{tabular}

RMSEA: Error medio cuadrático de aproximación. *No considera área "motora gruesa".

**No considera área "resolución de problemas". No se incluyeron valores de 60 meses

por muestra pequeña.

Tabla 5. Comparativo de puntos de corte del ASQ-3 en intervalo de D.E. según lugar del estudio, área de evaluación e intervalo de edad.

\begin{tabular}{|c|c|c|c|c|c|c|c|c|c|c|c|c|c|c|c|c|c|c|c|c|c|c|}
\hline Cuestionario (meses) & & 2 & 4 & 6 & 8 & 9 & 10 & 12 & 14 & 16 & 18 & 20 & 22 & 24 & 27 & 30 & 33 & 36 & 42 & 48 & 54 & 60 \\
\hline Área de evaluación & Lugar & \multicolumn{21}{|c|}{ Intervalos de punto de corte entre 1-2 Desviaciones estandar (D.E.) } \\
\hline \multirow[t]{3}{*}{ Comunicación } & Lima norte & 43.1-33.8 & $48.5-42.3$ & 44.7-37.4 & $47.5-41$ & $37.5-26.4$ & 42.9-33.9 & $43.8-34.9$ & $39.7-29.2$ & 30.7-18.4 & 23.8-9.6 & 25.6-10.2 & $30.5-16.1$ & $34.8-21$ & $40.8-30.2$ & 41.3-30.8 & $42.4-32.2$ & 45.7-37.4 & $49.2-43.3$ & $47.5-40.3$ & $52.6-47.3$ & 45.3-37.4 \\
\hline & Argentina & $39-30$ & $40-29$ & $44-36$ & $47-40$ & $44-36$ & $40-30$ & $26-10$ & $35-23$ & $27-17$ & $19-3$ & 23-7 & $21-4$ & $33-21$ & 30-14 & $39-28$ & 28-11 & $32-18$ & $33-25$ & $33-22$ & $34-20$ & $32-20$ \\
\hline & EEUU & $35.1-22.8$ & $43.4-34.6$ & $39.1-29.7$ & $42.6-33.1$ & 30-14 & $35.4-22.9$ & $30-15.6$ & $31.5-17.4$ & $30.5-16.8$ & 29.8-13.1 & $34.3-20.5$ & 29.9-13 & 38.1-25.2 & $37.2-24$ & 43.5-33.3 & $37.3-25.4$ & $41.5-31$ & $38.5-27.1$ & $41.8-30.7$ & $42.8-31.9$ & $42.8-33.2$ \\
\hline \multirow[t]{3}{*}{ Motor Grueso } & Lima norte & $48.8-42.4$ & $44-35.1$ & $27.6-14.7$ & $32.2-19.3$ & $22.4-7.3$ & $30.4-15.9$ & $31.2-16$ & $35.9-21.3$ & $48.6-41.2$ & $48-40.9$ & $47.8-40.8$ & $42.5-33.8$ & 43.1-33.7 & $41.7-31.7$ & $43-33.8$ & $43.3-32.8$ & $48.2-41$ & $49.9-43.9$ & $45.1-36.8$ & $46.4-38.9$ & 43-34-3 \\
\hline & Argentina & $37-23$ & $43-35$ & $34-22$ & $38-26$ & $40-31$ & $43-33$ & $24-7$ & $34-19$ & $43-33$ & $41-29$ & $33-16$ & $40-30$ & $45-38$ & $37-25$ & $43-37$ & $47-40$ & $44-36$ & $46-39$ & $29-21$ & $47-40$ & $45-39$ \\
\hline & EEUU & $48.4-41.8$ & $46.4-38.4$ & $33.9-22.3$ & $41.3-30.6$ & $32.3-17.8$ & $41.4-30.1$ & $35.5-21.5$ & $39.4-25.8$ & 47.8-37.9 & $46.3-37.4$ & $47.7-39.9$ & $39.1-27.8$ & $46.2-38.1$ & $39.1-28$ & $44.7-36.1$ & $44-34.8$ & 45.837 & $45.2-36.3$ & 42.732 .8 & $44.6-35.2$ & 41.8-31.3 \\
\hline \multirow[t]{3}{*}{ Motor Fino } & Lima norte & $43.3-35$ & $45-36.6$ & $40.6-29.9$ & $44.9-35.8$ & $44.1-34.8$ & $46-37.1$ & $43-33.6$ & $35.3-23.8$ & $36.6-24.4$ & $39.8-29.7$ & $38.9-28.8$ & $40.7-31.7$ & $39.8-30.7$ & $33.1-22.2$ & $28-13$ & $29.4-14.9$ & $32.5-18.9$ & $37.8-26.8$ & $34-21.9$ & $37.6-26.6$ & $42.2-33.7$ \\
\hline & Argentina & 43-35 & $34-21$ & $40-30$ & $49-43$ & $35-23$ & $39-28$ & $26-10$ & $28-12$ & $42-33.3$ & $41-31$ & $37-26$ & $36-26$ & $33-25$ & $24-10$ & $37-27$ & $27-12$ & $37-27$ & $27-16$ & $39-26$ & $28-15$ & 26-0ct \\
\hline & EEUU & $39.7-30.2$ & $40.4-29.6$ & $37-25.1$ & $47.8-40.2$ & $41.7-31.3$ & $46.2-38$ & $42.3-34.5$ & 34.8-23.1 & 41.9-32 & $43.2-34.3$ & 44.3-36.1 & $39-29.6$ & 43.3-35.2 & 31.1-18.4 & $33-19.3$ & $27.2-12.3$ & 32.6-18.1 & $33.6-19.8$ & 30.615 .8 & $31.6-17.3$ & 39.1-26.5 \\
\hline \multirow[t]{3}{*}{ Resolución de Problemas } & Lima norte & $36-24.9$ & $46.5-38.5$ & $43.5-34.1$ & $48.8-42.1$ & $40.7-30.8$ & 45.837 .5 & $37.6-26.5$ & $36.6-25$ & $36.5-24.4$ & $32.2-19.6$ & $39.4-30.9$ & $39.2-29.3$ & $37.3-26.8$ & $40.7-30.8$ & $37.8-26.4$ & $39.1-27.4$ & $43.9-34.7$ & $44.8-36.5$ & $48-40.8$ & $50.4-44.8$ & $47.6-40.4$ \\
\hline & Argentina & $30-15$ & $37-26$ & $46-38$ & $51-46$ & $42-33$ & $47-39$ & $20-3$ & $34-21$ & $43-35$ & $32-20$ & $37-27$ & $34-23$ & $38-28$ & $35-24$ & $32-19$ & $28-12$ & $32-18$ & 31-17 & 28-14 & $25-11$ & $27-15$ \\
\hline & EEUU & $36.4-24.6$ & $44.2-35$ & $39.1-27.7$ & $45-36.2$ & $39.1-28.7$ & $42.2-32.5$ & $38.1-27.3$ & $34.6-22.6$ & 40.930 .5 & $35.7-25.7$ & $38.5-28.8$ & $39.1-29.3$ & $39.4-29.8$ & $38.6-27.6$ & $38.5-27.1$ & $38.6-26.9$ & $41.1-30.3$ & 39.7-28.1 & 4231.3 & $39.7-28.1$ & $41.3-30$ \\
\hline \multirow[t]{4}{*}{ Socio-Individual } & Lima norte & $42.9-34.5$ & $46.6-39.3$ & $43.2-33.8$ & $45.6-37.7$ & $35.1-23.4$ & $38.2-27.6$ & $34.3-21.6$ & $36.8-26$ & $36.4-25.2$ & $37.4-26.4$ & $34.8-24$ & $40-30.5$ & $35.3-23.9$ & $33.4-22.7$ & $36.4-25.9$ & $36.1-24.7$ & $40.6-31.6$ & $43.3-35.3$ & $42.8-33.5$ & $44.4-36.5$ & $43.1-35.2$ \\
\hline & Argentina & $39-31$ & $40-30$ & $40-31$ & $44-36$ & $36-26$ & $41-31$ & $24-8$ & $40-30$ & $42-34$ & $40-30$ & $37-25$ & $41-32$ & $36-25$ & $33-20$ & $39-29$ & $43-35$ & $32-20$ & $43-35$ & $32-18$ & $36-27$ & $41-31$ \\
\hline & EEUU & 42.1-33.7 & $42.4-33.2$ & $36.7-25.3$ & $44.4-35.8$ & $30.7-18.9$ & $38.3-27.3$ & $33.6-21.7$ & $35.6-23.2$ & 37.3-26.4 & $37.4-27.2$ & 42.6-33.4 & 40.3-30.1 & $41.2-31.5$ & $36.1-25.3$ & $41.9-32$ & $39.8-29$ & $44.1-35.3$ & 41.3-31.1 & 38.426 .6 & $42.5-32.3$ & 46.9-39.1 \\
\hline & EEUU $=E$ & $s(6,11,1$ & entina & & & & & & & & & & & & & & & & & & & \\
\hline
\end{tabular}

gran mayoría de casos resultaron favorables, sea por el método de Lawshe, por la $\mathrm{V}$ de Aiken o por ambos (tabla no adjunta); sin embargo, no hubo claridad en el enunciado en algunos de ellos. La única excepción fue el ítem 14 del cuestionario de 18 meses (área motora fina), pues a pesar de ser considerado esencial no hubo acuerdo en sus atributos de Suficiencia, Pertinencia y Claridad.

Validez de ítem: Para la mayoría de los cuestionarios entre los 2 meses y 60 meses, los valores mostraron una Correlación biserial ítem-test altos; indicando tener un buen poder discriminatorio por ítem $(r b>$ 0,30 ). Algunos ítems no alcanzaron estos valores debido a que las respuestas fueron posiblemente una constante. En el ítem 20 del mes 60 correspondiente al área "resolución de problemas", no se pudo determinar la $r b$ entre éste y el total de la escala debido a que todas las respuestas tuvieron 10 puntos (en cada uno de los 61 niños del mes 60).

Análisis factorial: En la tabla 4 se muestran valores de la Prueba de bondad de ajuste mediante el Índice RMSEA. Se observó que la estructura factorial del $A S Q-3$ para los cuestionarios de los meses 2 a
36 meses fueron adecuadas en sus 5 dimensiones o áreas (Índice RMSEA $<0,10$ ). Sin embargo, para los cuestionarios entre 42 y 54 meses, el Índice RMSEA sobrepasó levemente el umbral; por lo que, en estas edades la estructura de 5 áreas debería ser revisada. Para la edad de 60 meses no fue posible determinar la estructura factorial debido a que el tamaño de la muestra fue muy pequeño, 61 cuestionarios.

Puntos de corte: En la tabla 5, se muestra de manera comparativa los valores de puntos de corte del ASQ-3 para la población de estudio. Con ello se determinó las zonas de riesgo para cada una de las 5 áreas de evaluación en los 21 cuestionarios. A las cifras obtenidas se adjuntan de manera comparativa las reportadas por Squires, J. et Al. $A S Q-3$ (EE. UU) $(11,12)$ y las obtenidas por Romero-Otalvaro A. et Al. (Buenos Aires-Argentina) (17).

\section{DISCUSIÓN}

El cuestionario ASQ-3 construido, validado y estandarizado inicialmente en EE.UU. fue traducido al español, francés y comercializado desde el 2009 $(6,11,12,20,21)$. Desde entonces fue adaptado y 
Adaptación, validación y puntos de corte del Cuestionario de edades y etapas-3ra edición (ASQ-3) en español Lima-Perú.

Tabla 6. Comparativo de Indicadores de validez y confiabilidad del ASQ-3 por autores.

\begin{tabular}{|c|c|c|c|c|c|}
\hline $\begin{array}{c}\text { Autor- año/ tamaño muestral/ } \\
\text { país }\end{array}$ & $\begin{array}{l}\text { Rango de } \\
\text { edad } \\
\text { (meses) }\end{array}$ & $\begin{array}{l}\text { Validez de } \\
\text { Ítem (rb) }\end{array}$ & $\begin{array}{l}\text { Coeficiente } \\
\text { alfa }\end{array}$ & S, E $(\%)$ & $\begin{array}{l}\text { Puntos de } \\
\text { corte } \\
\text { (meses) }\end{array}$ \\
\hline $\begin{array}{l}\text { Gudiel A. } 2020 ; n=5830 \\
\text { Zona urbana, Lima-Perú. }\end{array}$ & $2-60$ & $\mathrm{rb}>0,30$ & $\begin{array}{c}0,70-0,94 \\
(*)\end{array}$ & 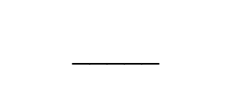 & $2-60$ \\
\hline $\begin{array}{l}\text { Squires J. 2009; } \mathrm{n}=18582 \\
\text { Oregón-USA }(11,12) .\end{array}$ & $2-60$ & 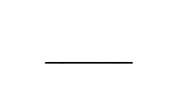 & $0,51-0,87$ & $\mathrm{~S}: 0,8 ; \mathrm{E}: 0,85$ & $2-60$ \\
\hline $\begin{array}{l}\text { Schonhaut L. 2013; } n=306 \\
\text { Santiago, Chile (24). }\end{array}$ & $8-30$ & & - & S:0,7; E:0,81 & - \\
\hline $\begin{array}{l}\text { Romero A. 2018; } n=630 \\
\text { Buenos Aires- Argentina (17) }\end{array}$ & $2-60$ & . & ـ & S:0,88; E: 0,94 & $2-60$ \\
\hline $\begin{array}{l}\text { Caridad-Araujo M. 2019; n=1 } \\
493 \\
\text { Zona rural, Perú (18). }\end{array}$ & $1-42$ & - & $\begin{array}{c}0.26 \\
-0.55(* *) \\
0.60 \\
-0.70(* * *)\end{array}$ & - & \\
\hline $\begin{array}{l}\text { Armijo I. 2015; } n=1572 \\
\text { Santiago-Chile (16). }\end{array}$ & $8-18$ & - & $0.66-0.85$ & ـ & \\
\hline $\begin{array}{l}\text { Filgueiras A. 2013; n=45 } 640 \\
\text { Brasil (14). }\end{array}$ & $6-60$ & $\mathrm{rb}>0,30$ & $0,52-0,89$ & 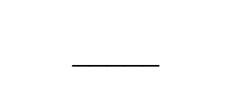 & \\
\hline $\begin{array}{l}\text { Rubio-Codina M. 2016; n=770 } \\
\text { Bogotá-Colombia (15). }\end{array}$ & $6-42$ & & $<0,55$ & $\begin{array}{l}\text { V. Concurrente } \\
\text { ( } r<0,4 \text { con } \\
\text { Bayley-III })\end{array}$ & - \\
\hline $\begin{array}{l}\text { Ortiz-León S. 2018; n=1 } 052 \\
\text { México (13). }\end{array}$ & $33-60$ & - & $0,77-0,88$ & . & \\
\hline $\begin{array}{l}\text { Xiao-Yang B. 2010; } \mathrm{n}=8472 \\
\text { Shangai-China (34). }\end{array}$ & $3-60$ & $\longrightarrow$ & 0,77 & S:0,85; E: 0,84 & $3-60$ \\
\hline $\begin{array}{l}\text { Van Heerden A. 2017; } n=853 \\
\text { Sudáfrica- Zambia (35). }\end{array}$ & $2-60$ & $\mathrm{rb}>0,30$ & $0,60-0,88$ & - & - \\
\hline
\end{tabular}

(*): Alfa ordinal, $\mathrm{r}=$ Coeficiente de correlación, $\mathrm{rb}=$ Correlación biserial, $\mathrm{m}=$ Meses, $\mathrm{V}=$ Validez, $\mathrm{S}=$ Sensibilidad, $\mathrm{E}=\mathrm{Especificidad}$. (**): Alfa de Cronbach del ASQ-3 de 6 ítems, en línea de base del estudio. (***): Alfa de Cronbach

del ASQ-3 de 9 ítems en línea de base del estudio. Los estudios acá referidos no indicaron valores de análisis factorial. En nuestro estudio la Prueba de bondad de ajuste del análisis factorial fue adecuado para los meses 2-36.

validado en muchos países del mundo incluido Latinoamérica (13-17). A la fecha fue traducido a más de 65 idiomas (26). En el proceso de validación de este instrumento para la población de nuestro estudio se cumplió en realizar la adaptación lingüística-cultural basado en las recomendaciones del editor y de la autora de la prueba original. Debido a su extensión no se incluyó en el presente informe los detalles de los ítems adaptados. En general la prueba tuvo pocos ítems que demandaron cambios importantes, resultando ser comprensible, práctico y pertinente en general.

Los indicadores de confiabilidad y validez del presente estudio hallados en rangos de aceptables a buenos, permitió establecer de manera general que la versión en español del ASQ-3 es un instrumento de vigilancia y tamizaje del desarrollo confiable y válido para el entorno y población donde se llevó a cabo el estudio. Si bien se tuvo buen número de tamaño de muestra, mayor de 200 para cada uno de 19 cuestionarios (entre 2 a 48 meses).

Para los intervalos de edad entre 14 a 36 meses el tamaño de muestra fue buena llegando a un promedio de 1333 cuestionarios por rango de edad. Sin embargo, para los 2 últimos cuestionarios (54 y 60 meses) sumaron 129 cuestionarios. Esto afectaría los resultados en la evaluación de los indicadores de validez principalmente del análisis factorial en esos grupos de edad. Esta diferencia posiblemente se deba a que los 2 primeros años se aplicaron los cuestionarios hasta los 36 meses, que luego se amplió hasta los 60 
meses. Típicamente una muestra se considera "buena" si se aplican $\geq 200$ cuestionarios por intervalo de edad y "adecuado" si es 50-100 (31).

Es de destacar que cerca de la mitad de las encuestas aplicadas en el presente estudio (45.7\%) correspondieron a cuestionarios con intervalos de edad entre 14 a 36 meses. Al respecto, la AAP y "Bright futures" recomiendan realizar una vigilancia y evaluación del desarrollo a los 9, 18 y 24 ó 30 meses de edad $(8,9)$; debido a que en estas estas edades transcurren etapas importantes del desarrollo infantil y a la vez se pueden identificar de manera confiable alteraciones de tipo sensorial, motora gruesa y fina, retrasos en las áreas social, lenguaje y cognitivo. Mientras que el tamizaje del trastorno del espectro autista se recomienda realizar a los 18 y 24 meses $(9,25)$

Los cuestionarios aplicados a padres o cuidadores como un instrumento de vigilancia y tamizaje del desarrollo, si bien poseen limitaciones en términos de posible sesgo de recuerdo y del conocimiento del curso evolutivo de sus niños, son considerados de manera general como una fuente confiable y relevante de información sobre el desarrollo infantil. Este tipo de pruebas si bien no establecen diagnóstico, pueden indicar si el niño transcurre un curso típico de desarrollo o tener riesgo de posible retraso o trastorno del desarrollo que justifique una evaluación más objetiva $(4,5,9,32,33)$.

Lipkin et al. reportan de un seguimiento de encuestas a pediatras afiliados a la AAP del 2002 al 2016, hallando que el uso de al menos un instrumento de tamizaje del desarrollo para niños $<36$ meses de edad se había triplicado en el transcurso del tiempo, de $23 \%$ en el 2002 a $63 \%$ en el $2016(7,9)$. El mismo estudio indicó que el ASQ-3 fue la herramienta de tamizaje más preferida en uso, $7 \%$ en el $2002,22 \%$ en el 2009 y $48 \%$ para el 2016; mientras que el Test de Denver-II y el PEDS no superaron 18\% para el año 2016, respectivamente.

Del análisis de confiabilidad por la consistencia interna de la prueba en el presente estudio, con indicadores de sus valores alfa entre 0,70- 0,94 (aceptables a muy buenos) entre el área del desarrollo y el puntaje general, asemejándose a lo indicado por Squires et al., $(6,11,12)$ del estudio original: 0,51 a 0,87 . Sin embargo, en ambos estudios no se examinó la relación entre los ítems y el área de desarrollo, tal como se muestra en la tabla 6 , estudios de validación en México (13), Brasil (14), Chile (16), China (34) y Sudáfrica (35) corroboran estos hallazgos.

El estudio original determinó validez de contenido mediante opinión de expertos con pediatras, pediatras del desarrollo y profesionales relacionados que laboraban con niños y hablaban español, señalando que revisaron la versión ASQ-2 en español para desarrollar el ASQ-3, aunque no se menciona un índice de acuerdo al respecto $(6,20)$. Para nuestro estudio, el Índice de validez de contenido fue adecuado indicando que hubo un buen acuerdo entre jueces para evaluar los atributos de la prueba en Suficiencia, Pertinencia y Claridad. Además, la validez de contenido medido por la Correlación biserial item-test fue alto para la mayoría de los ítems, indicando un buen poder discriminatorio de la prueba por ítem. Si bien algunos ítems no alcanzaron estos valores, posiblemente se debió a que las respuestas fueron constantes en unas y escasas en otras. Filgueiras en Brasil (14) y Van Heerden en Sudáfrica (35), reportaron hallazgos semejantes en estos indicadores.

El análisis factorial nos permitió establecer como válido al constructo "desarrollo psicomotor" representado en el ASQ-3, debido a que las 5 áreas o factores que lo constituyen estuvieron adecuadamente representados, con indicadores estadísticamente significativos para los cuestionarios de 2 a 36 meses, aunque para los cuestionarios de 42 a 60 meses la estructura de 5 áreas no alcanzó los valores adecuados, posiblemente relacionados al tamaño de la muestra. No obstante, este aspecto del análisis constituyó una de las principales fortalezas del estudio por su significado en el proceso de validación de una prueba.

Si bien no se reportó indicadores de análisis factorial en la validación original del ASQ-3; Moodie, S. et Al. (20) del Departamento de Salud y Servicios Humanos de los Estados Unidos (USHHS), señaló en un reporte del 2014, que el ASQ-3 del estudio original "posee una fuerte validez de constructo".

Para nuestro medio existe escasa información relacionada a la validación del ASQ-3 en un entorno urbano de consulta ambulatoria de pediatría. Tarazona D. (19), en un estudio local de validez concurrente entre el $A S Q-3$ y el Inventario del Desarrollo BattelleII (IDB-II), tomando a esta última como prueba referente, de una muestra de 129 evaluaciones (60 en Lima y 69 en Huamanga-Ayacucho-Perú); para las edades de 24 a 44 meses, halló una correlación (r) de Pearson de leve a moderada $(0,24-0,63)$. La 
Adaptación, validación y puntos de corte del Cuestionario de edades y etapas-3ra edición (ASQ-3) en español Lima-Perú.

consistencia interna general fue aceptable (Alfa de Cronbach: 0,77). Sin embargo, del análisis para cada área los valores fueron no deseables o inaceptables $(0,17-0,63)$. Estas observaciones posiblemente se deban a un pequeño número de la muestra.

Kyerematen V. et al. (36), empleando el $A S Q-2$ $(\mathrm{n}=77)$ y $A S Q-3(\mathrm{n}=52)$ en un estudio de aplicación exploratoria en una población infantil de bajos ingresos, zona suburbana de Lima, señaló una posible relación entre los bajos puntajes obtenidos con el $A S Q$ y el estado de desnutrición de los niños sugiriendo que tal vez se debió a enfermedades diarreicas. Sin embargo, no se reportaron indicadores de confiabilidad y validez del ASQ.

Caridad-Araujo M. et al. (18), en un estudio diseñado para evaluar el impacto de un programa social en 12 departamentos del Perú (zonas rurales predominantemente andino-amazónicas), para los años 2013 a 2015, aplicando versiones del ASQ3 adaptados de 6 y 9 ítems por área (no se indicó el contenido de los 3 ítems adicionales) a una muestra de 1493 padres de niños (ASQ-3) y a 385 niños mediante la Escala de Bayley-III, menores de 42 meses; la consistencia interna alcanzada por el Coeficiente alfa de Cronbach para el ASQ-3 de 6 ítems por área fue < 0.7, mientras que para el ASQ-3 de 9 ítems fue algo mejor (0.60-0.70).

De las pruebas de tamizaje o de evaluación del desarrollo en la infancia de uso común en nuestro medio, como la Escala de Evaluación del Desarrollo Psicomotor 0 a 24 meses (EEDP), Test de Desarrollo Psicomotor 2 a 5 años (TEPSI), Test Peruano de Evaluación del Desarrollo del niño 0 a 30 meses (37), Escala del desarrollo infantil- ESDI (38), Inventario de desarrollo Battelle-tamizaje (39) y la Escala del desarrollo infantil Bayley-III- tamizaje (40), requieren todas de personal entrenado para su aplicación, mientras que los cuestionarios como el ASQ-3 no requieren de personal sanitario entrenado, son los padres que lo completan $(6,11,32)$.

El haber desarrollado la validación del ASQ3 en un ámbito urbano no permite extrapolar su aplicabilidad para una zona suburbana o rural. No se llegó a determinar la sensibilidad, especificidad y valor predictivo de la prueba debido a que no fue parte de los objetivos del estudio.

Se concluyó para nuestro estudio que el ASQ-3 en su versión en español, es un instrumento válido y confiable para ser empleado en la vigilancia del desarrollo psicomotor como cuestionario aplicado a padres de niños entre 1 a 66 meses en un entorno urbano de consulta ambulatoria.

Declaración de Financiamiento y de Conflictos de Intereses.

Los autores declaramos no tener conflictos de interés, sean estos de orden económico, institucional, laboral o personal relevante para este artículo. Este estudio fue autofinanciado por los autores y no contiene discusión de un uso no aprobado/ investigativo de un producto/ dispositivo comercial.

Agradecimiento: Los autores agradecen a los padres que participaron en el estudio a través del llenado de los cuestionarios. Al personal asistencial y administrativo de la Clínica Mundo Salud lugar donde se llevó a cabo el estudio. A Óscar Ortíz A. por su invaluable ayuda en el procesamiento estadístico. A los licenciados July Paredes L., Fernando Vergel P., Liliana Lovatón G., Yesenia Bozeta Q., por su participación en el proceso de Adaptación lingüística y cultural del ASQ-3.

\section{Correspondencia:}

Adriel Gudiel Hermoza,

Av. Carlos Izaguirre 1234, Los Olivos; código postal Lima-39, teléfono: 989011375

Correo electrónico: adrielgudielh@gmail.com

\section{REFERENCIAS BIBLIOGRAFICAS}

1. Johnson CP, Blasco P. Infant Growth and Development. Pediatrics in Review. 1997; 18 (7): 224-242.

2. Scharf RJ, Scharf GJ, Stroustrup A. Developmental Milestones. Pediatrics in Review. 2016; 37;25-38.

3. Macias MM, Lipkin PH. Developmental and behavioral surveillance and screening within the medical home. En: Voigt RG, Macías MM, Myers SM, Tapia CD. Editores. American academy of pediatrics. Developmental and behavioral pediatrics. 2da edición. Illinois, USA: AAP; 2018.p. 135-163.

4. Marks KP, La Rosa AC. Understanding developmental- behavioral screening measures. Pediatr Rev. 2012;33 (10):448-458.

5. Drotar D, Stancin T, Dworkin PH, Sices L, Wood S. Selecting developmental surveillance and screening tools. Pediatr Rev. 2008;29 (10): e52-e58.

6. Squires J, Bricker D. Ages \& Stages Questionnaires: Third edition (ASQ-3), in Spanish. Baltimore, MD. USA: Paul H. Brookes Publishing Co Inc.; 2009. 
7. Lipkin PH, Macias M., Baer-Chen B, et al. Trends in pediatricians' developmental screening: 20022016. Pediatrics. 2020;145(4): e20190851.

8. Council on Children with Disabilities, Section on Developmental Behavioral Pediatrics, Bright Futures Steering Committee, Medical Home Initiatives for Children with Special Needs Project Advisory Committee. Identifying infants and young children with developmental disorders in the Medical Home: An algorithm for developmental surveillance and screening. Pediatrics. 2006; 118:405- 420.

9. Lipkin PH, Macias M. AAP Council on Children with Disabilities, Section on Developmental and Behavioral Pediatrics. Promoting optimal development: Identifying infants and young children with developmental disorders through developmental surveillance and screening. Pediatrics. 2020;145(1): e20193449.

10. Fiszbein A, Guerrero G, Rojas V. Medición del Desarrollo Infantil en América Latina: Construyendo una agenda regional. Inter-American Dialogue \& GRADE. The dialogue. 2016: 1-11.

11. Squires J, Twombly E, Bricker D, Potter L. ASQ-3 User's guide. Inc. Baltimore, MD. USA: Paul H. Brookes publishing Co; 2009.

12. Squires J, Twombly E, Bricker D, Potter LW. Psychometric studies of ASQ, third edition. Excerpted from: ASQ-3 User's Guide. Baltimore, MD.USA: Paul H. Brookes publishing Co; 2009.

13. Ortiz-León S, Granados-Rojas A, Cavazos-Olivo J, et al. Internal and inter-rater reliability of the ASQ-3 in Mexican preschoolers. Salud Mental. 2018;41(2): 65-72.

14. Filgueiras A, Pires P, Maissonette S, LandeiraFernandez J. Psychometric properties of the Brazilian-adapted version of the Ages and Stages Questionnaire in public child daycare centers. Early Hum Dev. 2013;89(8):561-576.

15. Rubio-Codina M, Caridad-Araujo M, Attanasio O, Grantham-McGregor S. Concurrent validity and feasibility of short tests currently used to measure early childhood development in large scale studies: Methodology and results. Washington DC: Inter-American Development Bank (IDB); 2016.

16. Armijo I, Schonhaut L, Cordero M. Validation of the Chilean version of the Ages and Stages Questionnaire (ASQ-CL) in community health settings. Early Hum Dev. 2015; 91(12): 671-6.

17. Romero-Otalvaro A, Grañana N, Gaeto N, et al. ASQ3: Validación del Cuestionario de Edades y Etapas para la detección de trastornos del neurodesarrollo en niños argentinos. Arch Argent Pediatr. 2018;116 (1):7-13.

18. Caridad-Araujo M, Dormal M, Lazarte F, Oré B, Rubio-Codina M. Adaptación, validación $\mathrm{y}$ propiedades psicométricas del ASQ-3 y del Bayley-
III en niños menores de 42 meses de zonas rurales de Perú. Banco Interamericano de Desarrollo. Nota técnica del BID $N^{\circ} 1685$. Washington DC: Banco Interamericano de Desarrollo; 2019.

19. Tarazona CD, Campos S M. Estudio de validez concurrente entre una versión culturalmente adaptada del Age and Stages Questionnaire-3 y la versión completa del Battelle developmental inventory en una muestra de niños peruanos de 24 a 44 meses. DGSE-MIDIS. 2014;1: 1-6.

20. Moodie S, Daneri P, Goldhagen S, Halle T, Green K, LaMonte L. Early childhood developmental screening: A compendium of measures for children ages birth to five (OPRE Report 2014 $\neg 11$ ). Washington DC: Office of Planning, Research and Evaluation, Administration for Children and Families, U.S. Department of Health and Human Services; 2014.

21. Singha A, Jung-Yehb C, Boone-Blanchard S. Ages and Stages Questionnaire: a global screening scale. Bol Med Hosp Infant Mex. 2017;74(1):5-12.

22. Schonhaut L, Armijo I. Aplicabilidad del Ages \& Stages Questionnaires para el tamizaje del desarrollo psicomotor. Rev Chil Pediatr. 2014; 85 (1):12-21.

23. Romero A, Nereida M. Instrumentos de evaluación de pesquisa de neurodesarrollo en la intervención temprana. Tesis Psicológica. 2016;11(2):54-71.

24. Schonhaut L, Armijo I, Schönstedt M, Alvarez J, Cordero M. Validity of the Ages and Stages Questionnaires in Term and Preterm Infants. Pediatrics. 2013;131: e1468-e1463.

25. Beacham C, Reid M, Bradshaw J, et al. Screening for autism spectrum disorder. Profiles of children who are missed. J Dev Behav Pediatr. 2018; 39 (9): 673682.

26. Brookes publishing Co. Ages \& Stages Questionnaires Guidelines for cultural and linguistic adaptation of ASQ-3 and ASQ:SE-2. Baltimore, MD.USA: Paul H. Brookes publishing Co; 2017. (Citado el 15 de junio 2018). Disponible en: http://archive.brookes publishing.com/documents/ASQ-cultural-andlinguistic-adaptation. pdf

27. Zumbo BD, Gadermann AM, Zeisser C. Ordinal versions of coefficients alpha and theta for likert rating scales. J Mod Appl Stat Methods. 2007; 6 (1):21-29.

28. Lawshe $\mathrm{CH}$. A quantitative approach to content validity. Personnel psychology. 1975; 28 (4):563575.

29. Tristán-López A. Modificación al modelo de Lawshe para el dictamen cuantitativo de la validez de contenido de un instrumento objetivo. Avances en Medición. 2008; 6:37-48.

30. Aryanto B, Hariono A, Pahalawidi C. Construct validity for talent identification test athletic with Aiken's V. Advances in Social Science, Education and Humanities Research. 2018; 278: 647-649. 
Adaptación, validación y puntos de corte del Cuestionario de edades y etapas-3ra edición (ASQ-3) en español Lima-Perú.

31. Marks KP, La Rosa AC. Supplementary data. What properties make a screening tool psychometrically sound? In: Marks KP, LaRosa AC. Understanding developmental-behavioral screening measures. Pediatr Rev. 2012; 33 (10): 448-458.

32. Squires J. Utilidad de padres y cuidadores como evaluadores certeros del desarrollo en niños menores. Rev Chil Pediatr. 2017; 88(1):22-24.

33. Glascoe FP. Using parents' concerns to detect and address developmental and behavioral problems. JSPN. 1999; 4 (1): 24-35.

34. Xiao-yan B, Guo-ying Y, Squires J, Mei W, Ching-I C, Bing-hua F. Studies of the norm and psychometric properties of Ages and Stages Questionnaires in Shanghai children. Zhonghua er ke za zhi. 2010; 48:492-6.

35. Van-Heerden A, Hsiao C, Matafwali B, Louw J, Richter L. Support for the feasibility of the ages and stages questionnaire as a developmental screening tool: a cross-sectional study of South African and Zambian children aged 2-60 months. BMC Pediatr. 2017; 17:55.

36. Kyerematen V, Hamb A, Oberhelman RA, Cabrera L, Bernabe-Ortiz A, Berry S.J. Exploratory application of the Ages and Stages (ASQ) child development screening test in a low-income Peruvian shantytown population. BMJ Open. 2014;4(1): e4132-4.
37. Ministerio de Salud. Norma técnica de salud para el control de crecimiento y desarrollo de la niña y el niño menor de cinco años. Lima: Ministerio de Salud;2 011. (Citado el 15 de octubre 2019). Disponible en: http://www.redsaludcce.gob.pe/ Modernidad/archivos/dais/ppan/normast/ CRED.pdf. 38. Ministerio de Desarrollo e Inclusión Social. Orientaciones para la aplicación de la Escala de desarrollo infantil - ESDI en el Programa nacional Cuna más. Edic. Gráfica técnica SRL. Lima. Ministerio de Desarrollo e Inclusión Social. Lima: Ministerio de Desarrollo e Inclusión Social; 2016. (Citado el 15 de octubre 2019). Disponible en: https:// es.scribd.com/document/354519560/OrientacionesPara-La-Aplicacion-de-La-ESDI-en-El-PNCM.

39. Newborg J, Stock JR, Wnek L. Inventario de Desarrollo Batttelle: Manual de aplicación. Madrid: Ediciones TEA; 2011.

40. Bayley N, Aylward GP. Bayley Scales of Infant and Toddler Development Screening Test, Third Edition. Bayley-III Screening test. Pearson; 2006. (Citado el 15 junio del 2018). Disponible en: https://www. pearsonassessments.com/store/usassessments/ en/Store/Profe ssional-Assessments/Cognition-\%26Neuro/Brief/Bayley-Scales-of-Infant-andToddler-Development-Screening-Test-\%7C-ThirdEdition/p/100000108.html.

Recibido: 14/08/2020

Aceptado: 02/06/2021 